\title{
OPEN A Mendelian randomization analysis of the relationship between cardioembolic risk factors and ischemic stroke
}

\author{
Danyang Tian ${ }^{1}$, Linjing Zhang ${ }^{1}$, Zhenhuang Zhuang ${ }^{2}$, Tao Huang ${ }^{2,3 凶}$ \& Dongsheng Fan ${ }^{1,3 \bowtie ~}$
}

Observational studies have shown that several risk factors are associated with cardioembolic stroke. However, whether such associations reflect causality remains unknown. We aimed to determine whether established and provisional cardioembolic risk factors are causally associated with cardioembolic stroke. Genetic instruments for atrial fibrillation (AF), myocardial infarction (MI), electrocardiogram (ECG) indices and N-terminal pro-brain natriuretic peptide (NT-pro BNP) were obtained from large genetic consortiums. Summarized data of ischemic stroke and its subtypes were extracted from the MEGASTROKE consortium. Causal estimates were calculated by applying inverse-variance weighted analysis, weighted median analysis, simple median analysis and Mendelian randomization (MR)-Egger regression. Genetically predicted AF was significantly associated with higher odds of ischemic stroke (odds ratio (OR): 1.20, 95\% confidence intervals (CI): 1.16-1.24, $\mathrm{P}=6.53 \times 10^{-30}$ ) and cardioembolic stroke (OR: $1.95,95 \% \mathrm{Cl}: 1.85-2.06, \mathrm{P}=8.81 \times 10^{-125}$ ). Suggestive associations were found between genetically determined resting heart rate and higher odds of ischemic stroke (OR: $1.01,95 \% \mathrm{Cl}: 1.00-1.02, \mathrm{P}=0.005)$, large-artery atherosclerotic stroke (OR: 1.02, 95\% Cl: 1.00-1.04, $\mathrm{P}=0.026$ ) and cardioembolic stroke (OR: $1.02,95 \% \mathrm{Cl}: 1.00-1.04, \mathrm{P}=0.028$ ). There was no causal association of $\mathrm{P}$-wave terminal force in the precordial lead V1 (PTFVI), P-wave duration (PWD), NT-pro BNP or PR interval with ischemic stroke or any subtype.

Stroke leads to $10 \%$ of deaths worldwide and substantial long-term disability ${ }^{1}$. Cardioembolic stroke (CES) is disproportionately more disabling than nonembolic mechanisms of stroke and accounts for an increasing proportion of ischemic strokes and might multiply several-fold over the next decades in an aging society ${ }^{2,3}$. Furthermore, among the one-third of ischemic strokes that have no identifiable cause after standard evaluation, it is increasingly accepted that many arise from a distant embolism (cardioembolic in a large proportion) rather than in situ cerebrovascular disease. Given the heavy social and economic burden, understanding the underlying mechanisms and subsequently preventing CES are important.

Many studies have shed light on various mechanisms of suspected CES. A recent review reported that subclinical atrial fibrillation (AF), atrial cardiopathy, unrecognized myocardial infarction (MI) and patent foramen ovale are occult mechanisms inducing $\mathrm{CES}^{4}$. It is suspected that abnormal atrial substrate might form a nidus for thromboembolism before AF occurs ${ }^{5}$. Observational studies have shown associations between electrocardiographic (P-wave terminal force in lead V1 (PTFV1), P-wave duration (PWD) and maximum P-wave area $^{6}$, biochemical (N-terminal pro-brain natriuretic peptide (NT-pro BNP)) ${ }^{7}$, and mechanical (left atrial diameter ${ }^{8}$ indicators of left atrial dysfunction and ischemic stroke risk. However, the results have not always been consistent ${ }^{9}$. Resting heart rate (RHR) and PR interval prolongation have been reported to be associated with AF risk, which gave us a clue to investigate whether they are associated with $\mathrm{CES}^{10}$. Several observational studies and meta-analyses have shown relationships between RHR and ischemic stroke, while other studies have shown inconsistent results ${ }^{11}$. Furthermore, the previous observational studies did not specify ischemic stroke etiologies $^{12}$. Studies also show an association between PR interval prolongation and stroke. However, these studies contained a small number of cases. Meta-analysis of the relationship between PR interval and stroke showed

${ }^{1}$ Department of Neurology, Peking University Third Hospital, No. 49, North Garden Rd., Haidian District, Beijing 100191, China. '2Department of Epidemiology and Biostatistics, School of Public Health, Peking University, No. 38, Xueyuan Rd., Haidian District, Beijing 100191, China. ${ }^{3}$ These authors contributed equally: Tao Huang and Dongsheng Fan. ${ }^{\circledR}$ email: huangtaotao@pku.edu.cn; dsfan2010@aliyun.com 


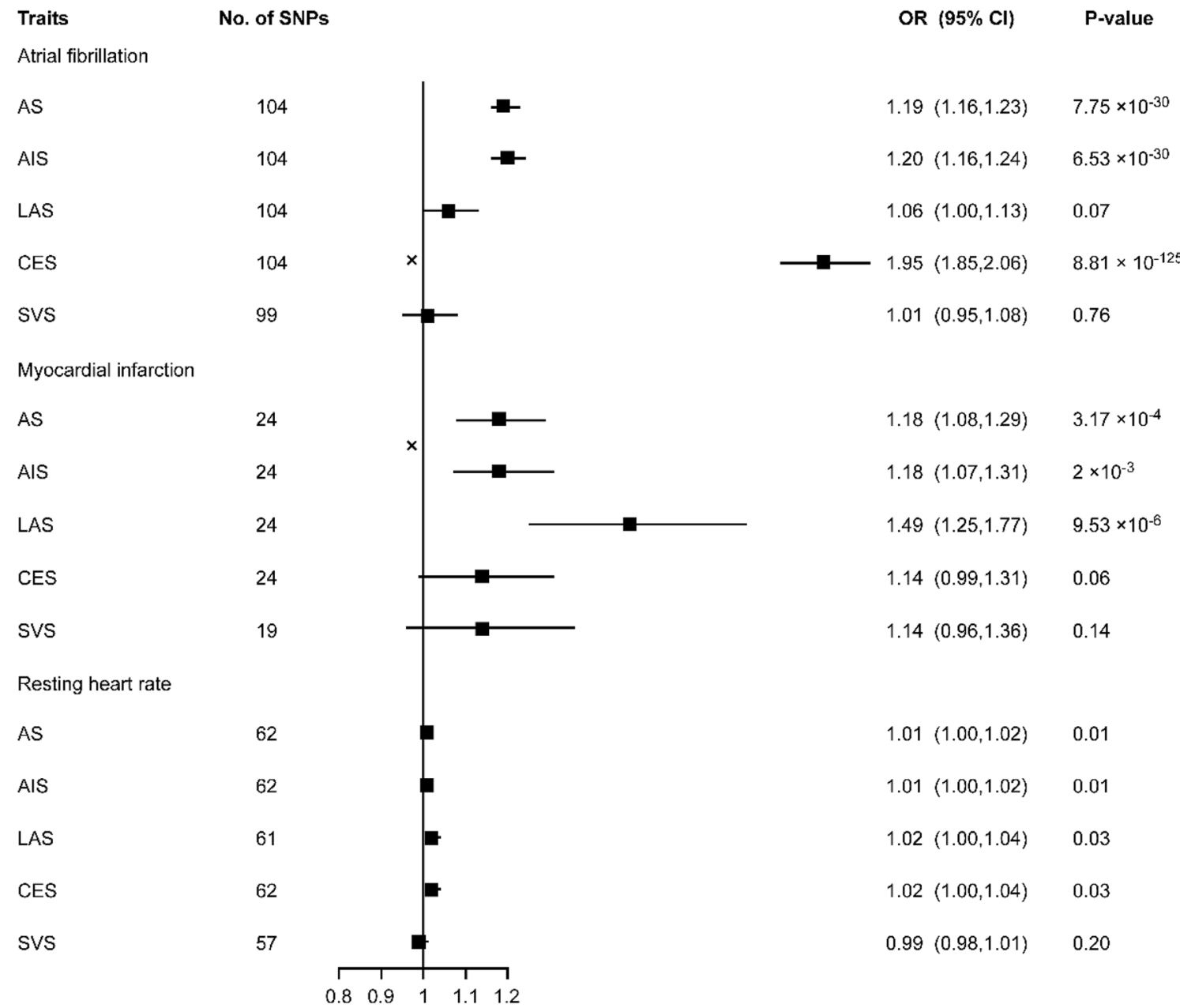

Figure 1. Mendelian randomization for associations between AF, MI, and RHR and stroke and its subtypes. $A F$ atrial fibrillation, $M I$ myocardial infarction, $R H R$ resting heart rate, SNP single-nucleotide polymorphism, $A S$ any stroke, AIS any ischemic stroke, LAS large artery atherosclerosis stroke, CES cardioembolic stroke, SVS small vessel stroke.

negative results ${ }^{13}$. It is worth noting that the conclusion from observational studies may be biased due to reverse causation or confounding. Therefore, the causality behind such associations remains largely unclear.

Mendelian randomization (MR) is a technique widely used to examine the causality relationships between risk factors and various diseases in the absence of pleiotropy ${ }^{14}$. During conception, the genetic alleles are randomly assorted, hence, it is less likely to be affected by confounding factors comparing with observational studies. In addition, since genotype is not affected by disease, it could avoid reverse causation bias. Since the limitation of reverse causation and cofounding in observational studies, we use MR in genetic perspective, which could overcome the limitations and investigate the causal risk factors of cardioembolic stroke, and provide basis for further prevention.

\section{Results}

Genetically predicted AF was associated with significantly higher odds of any stroke (OR: 1.19, 95\% CI: 1.16-1.23, $\mathrm{P}=7.74 \times 10^{-30}$ ), any ischemic stroke (OR: $1.20,95 \% \mathrm{CI}: 1.16-1.24, \mathrm{P}=6.53 \times 10^{-30}$ ) and CES (OR: $1.95,95 \% \mathrm{CI}$ : $1.85-2.06, \mathrm{P}=8.81 \times 10^{-125}$ ) but not with LAS (OR: $\left.1.06,95 \% \mathrm{CI}: 1.00-1.13, \mathrm{P}=0.07\right)$ or SVS (OR: $1.01,95 \%$ CI: $0.95-1.08, \mathrm{P}=0.76$ ). (Fig. 1) The results of the leave-one-out analysis showed that no single variant had an influence on the association between AF and stroke or its subtypes. The results from the MR-Egger regression analysis, the weighted median analysis and the simple median analysis were consistent with the IVW analysis (supplement table 2). MR-Egger regression analysis provided no evidence of directional pleiotropy for the associations of AF with any stroke (intercept $=0.002, \mathrm{p}=0.43$ ), any ischemic stroke (intercept $=0.002, \mathrm{p}=0.55)$ or CES (intercept $=-0.004, p=0.39$ ).

Genetically predicted MI was associated with significantly higher odds of any stroke (OR: 1.18, 95\% CI: $1.08-1.29, \mathrm{P}=3.00 \times 10^{-4}$ ) and LAS (OR: $\left.1.49,95 \% \mathrm{CI}: 1.25-1.77, \mathrm{P}=9.53 \times 10^{-6}\right)$. (Fig. 1 ) It was suggestively associated with any ischemic stroke (OR: $1.18,95 \% \mathrm{CI}: 1.07-1.31, \mathrm{P}=0.002)$ but not with CES (OR: $1.06,95 \%$ CI: $1.00-1.13, \mathrm{P}=0.06$ ) or SVS (OR: $1.01,95 \% \mathrm{CI}: 0.95-1.08, \mathrm{P}=0.76$ ). In the leave-one-out analysis, exclusion of rs1870634, rs2019090, rs2681472 rs58131196, rs1004467 or rs10176178 induced a significant result between MI 


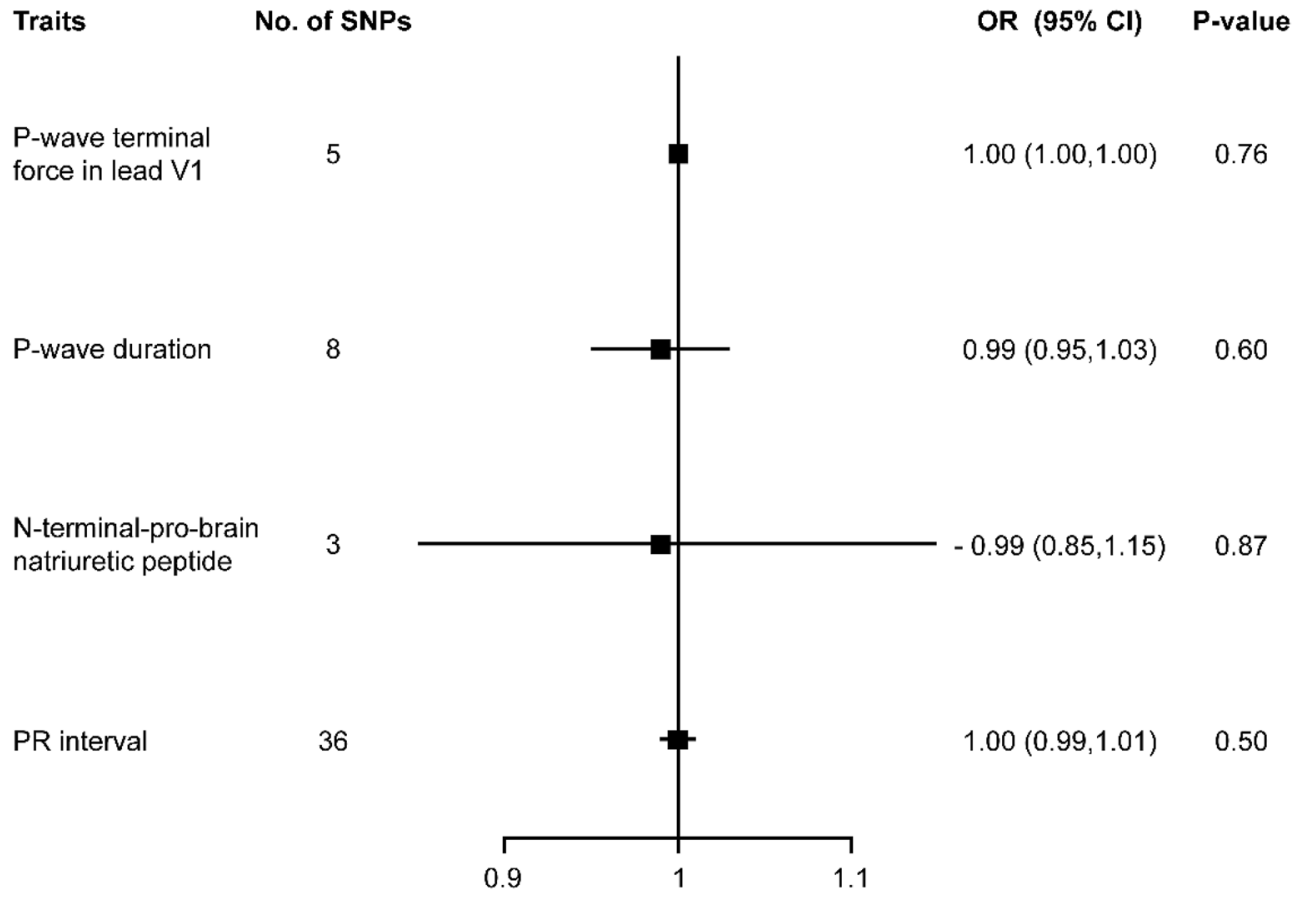

Figure 2. Mendelian randomization for associations between PTFVI, PWD, NT-pro BNP, and PR interval and CES. PTFVI P-wave terminal force in lead V1, PWD P-wave duration, CES cardioembolic stroke.

and CES. The results from the weighted median analysis and the simple median analysis were consistent with the IVW analysis (supplement table 2). MR-Egger regression analysis provided no evidence of directional pleiotropy for the associations of MI with any stroke (intercept $=-0.001, \mathrm{p}=0.84$ ), any ischemic stroke (intercept $=-0.005$, $\mathrm{p}=0.72$ ) or LAS (intercept $=-0.019, \mathrm{p}=0.40$ ).

A suggestively significant association was found between RHR and higher odds of any stroke (OR: 1.01, 95\% CI: $1.00-1.02, \mathrm{P}=0.009$ ), any ischemic stroke (OR: $1.01,95 \% \mathrm{CI}: 1.00-1.02, \mathrm{P}=0.005)$, LAS (OR: $1.02,95 \% \mathrm{CI}$ : $1.00-1.04, \mathrm{P}=0.026)$ and CES (OR: $1.02,95 \% \mathrm{CI}: 1.00-1.04, \mathrm{P}=0.028)$ but not with SVS (OR: $0.99,95 \% \mathrm{CI}$ : $0.98-1.01, \mathrm{P}=0.203$ ). (Fig. 1) The results of the leave-one-out analysis showed that no single variant had an influential influence on the association between RHR and stroke or its subtypes. The results from the simple median analysis for any stroke and CES and the weighted median analysis for any stroke were consistent with the IVW analysis (supplement table 2). MR-Egger regression analysis provided no evidence of directional pleiotropy for the associations of RHR with any stroke (intercept $=6.98 \times 10^{-4}, \mathrm{p}=0.84$ ), any ischemic stroke (intercept $=0.001$, $\mathrm{p}=0.75$ ), LAS (intercept $=-0.004, \mathrm{p}=0.66$ ) or CES (intercept $=0.004, \mathrm{p}=0.64$ ).

There was no association between PTFVI, PWD, NT-pro BNP or PR interval and strokes or any subtype (Fig. 2 and supplement table 2). The results were consistent with MR-Egger regression analysis, weighted median analysis and simple median analysis, and no single genetic variant had an influential influence in the leave-oneout analyses. All MR size effect, leave one out and scatter plot results were shown in supplement figures.

\section{Discussion}

The main findings from this study can be summarized as follows: (1) Higher odds of AF and increased RHR are causally associated with higher risk of CES; (2) We did not find evidence for a causal relationship between MI and the risk of CES unless rs1870634, rs2019090, rs2681472 rs58131196, rs1004467 or rs10176178 were excluded; (3) No evidence of causal relationship between previously observed cardioembolic risk factors such as PTFVI, PWD, NT-pro BNP or PR interval and risk of CES was found from a genetic perspective; 4. Genetically increased odds of MI and RHR are causally associated with higher risk of LAS. The present MR study explicitly examined cardioembolic-related traits while avoiding confounding and reverse causal bias.

The following implications for clinical practice are reasonable based on the main findings of this study: (1) To prevent CES and further reduce the social and economic burden, prevention of AF is an important part, which is consistent with observational studies. (2) Increasing RHR is a causal risk factor of CES, remind us to pay more attention on people with high RHR for the underlying CES burden. (3) We did not find the causal relationship between PTFVI, PWD, NT-pro BNP or PR interval and CES risk, more studies are needed, and discovering other indices for CES risk are needed.

$\mathrm{AF}$ is an established major risk factor for CES. This study provided causal evidence of an association between $\mathrm{AF}$ and CES from a genetic perspective. In addition to persistent/permanent AF, paroxysmal AF with an asymptomatic form may also cause a stroke but then revert back to sinus rhythm when the patient presents for evaluation. Detecting paroxysmal AF using prolonged heart rhythm monitoring is important to identify a stroke with 
cardioembolic origin ${ }^{15}$. Although AF is reported to be associated with atherosclerotic factors, we did not find a significant association of AF with LAS or SVS.

In observational studies, there is a high prevalence of documented stroke/transient ischemic attack (TIA) in patients with acute coronary syndromes $(\mathrm{ACS})^{16}$. The risk of stroke appears to be elevated for longer than 1 month after $\mathrm{MI}^{17}$. Recently, it was discovered that unrecognized MIs, which make up at least one-third of all MIs, may also be a risk factor for stroke ${ }^{18}$. In the present MR study, we found a causal relationship between MI and ischemic stroke. However, MI was not significantly associated with CES unless rs1870634, rs2019090, rs2681472 rs58131196, rs1004467 or rs10176178 were removed. MI was identified as a cardioembolic risk factor for stroke in previous observation studies. However, evidence from this MR analysis did not support this previous conclusion. We could not exclude the possibility that the null association was due to the limited power. Another explanation is that with a larger number of variants, the potential for pleiotropy is greater, which could have diluted the association in our analysis. MI and LAS shared common atherosclerosis factors, which might indicate an association between MI and LAS. This MR study supported that MI is causally associated with the risk of LAS $\left(\mathrm{P}=9.53 \times 10^{-6}\right)$.

The results from previous observational studies of the relationship between RHR and stroke have been inconsistent. One study has shown that when compared with patients with $\mathrm{RHR}<65$ beats/min, patients with RHR $>80$ beats/min had a $38 \%$ higher risk of ischemic stroke ${ }^{19}$. A meta-analysis showed that the summary relative risk (RR) per 10 beats per minute increase in RHR was 1.06 (95\% CI 1.02-1.10) for total stroke ${ }^{20}$. However, some other studies did not find an association between RHR and stroke ${ }^{11}$. Among previous studies, few further investigated RHR and ischemic stroke subtypes. The present MR study showed a suggested relationship between RHR and ischemic stroke, especially LAS and CES. The MR analysis could avoid confounding and reverse causal bias, which could provide evidence of causality between RHR and stroke despite the inconsistent results from observational studies. The possible mechanisms are as follows: (1) Heart rate reduction improves aortic compliance, which plays an important role in hypertension and cardiac autonomic rhythm; (2) Higher HR is related to sympathetic overactivity, which might reflect vessel stiffness, cardiac remodeling, and metabolic change and may also induce arrythmia; (3) Higher HR might induce oxidative stress, which is associated with endothelial dysfunction and promote atherosclerosis ${ }^{21}$.

Since cardioembolic sources are hard to detect in some cases, researchers are attempting to identify markers that suggest a cardioembolic source. The anomaly of the electrocardiographic P-wave represents abnormal atrial motion, which could induce increased atrial pressure and then electronic and mechanical remodeling ${ }^{22}$. The anomaly of electrocardiographic P-waves was a possible cardioembolic risk factor in previous observational studies. Previous observational studies showed inconsistent results regarding the relationship between P-wave indices (PWI) and stroke risk. A meta-analysis showed that both PTFVI and PWD were significantly associated with stroke risk. Unfortunately, the MR study did not find a relationship between PWI and stroke risk, which is consistent with some of the previous studies ${ }^{23,24}$. Observational studies have potential confounding factors and possible selection bias. Furthermore, we could not exclude the possibility that the present GWAS results ignored a weak association.

Previous studies have shown that patients with higher levels of NT-pro BNP have a ninefold increase in stroke risk than patients with lower NT-pro BNP levels ${ }^{25}$. In addition, increased NT-pro BNP levels are associated with AF risk. Even in the absence of AF, increased NT-pro BNP levels suggest atrial disorders, which contribute to stroke risk ${ }^{4}$. This MR study did not present a significant causal relationship between NT-pro BNP and stroke. We also could not exclude the possibility that the present GWAS results ignored a weak association. Further study is still needed.

A prolonged PR interval is thought to be associated with AF risk and pulse wave velocity, and the latter has been closely associated with the risk of $\mathrm{LAS}^{26}$. A prospective study showed that a PR interval $>200 \mathrm{~ms}$ was associated with increased stroke risk ${ }^{27}$. However, a meta-analysis showed that the PR interval was significantly correlated with AF and heart failure but not stroke ${ }^{13}$. This MR study did not find causality between the PR interval and risk of stroke.

The strengths of this study are listed below. First, the MR design technique avoids bias from reverse causation and generally reduces confounding by other modifiable environmental exposures. Second, the present study used data from large GWAS of the risk factors, which suggests strong statistical power. Third, we evaluated the causal effect of cardioembolic risk factors on ischemic stroke and its subtypes, illuminating the inconsistent results from observational studies. Fourth, we performed multiple sensitivity analyses and pleiotropy tests. These analyses showed consistent results, thus minimizing the possibility of bias in the MR analyses.

The study also has some limitations. First, the genetic variants associated with the traits explain only a small fraction of the variation in the risk factors, and we cannot exclude the possibility that the lack of significant associations of PTFV1, PWD, and NT-pro BNP with ischemic stroke subtypes was due to insufficient statistical power. Second, we did not obtain GWAS data for other cardioembolic risk factors, such as patent foramen ovale and left atrial diameter, because the data were unavailable, and further study is still needed. Third, we could not examine potential nonlinear relationships for the continuous risk factors. Finally, since the GWAS study of PR interval and NT-pro BNP were of European ethnicity, and other traits and the outcomes were transethnic, we could not avoid ethnic bias in the relationship between PR interval, NT-pro BNP and ischemic stroke.

In summary, this study provides genetic evidence that AF and RHR, but not MI may play causal roles in development of stroke, especially CES. Our findings further suggest that MI and RHR have causal relationships with LAS. PTFVI, PWD, NT-pro BNP or PR interval may not causally be associated with CES. 

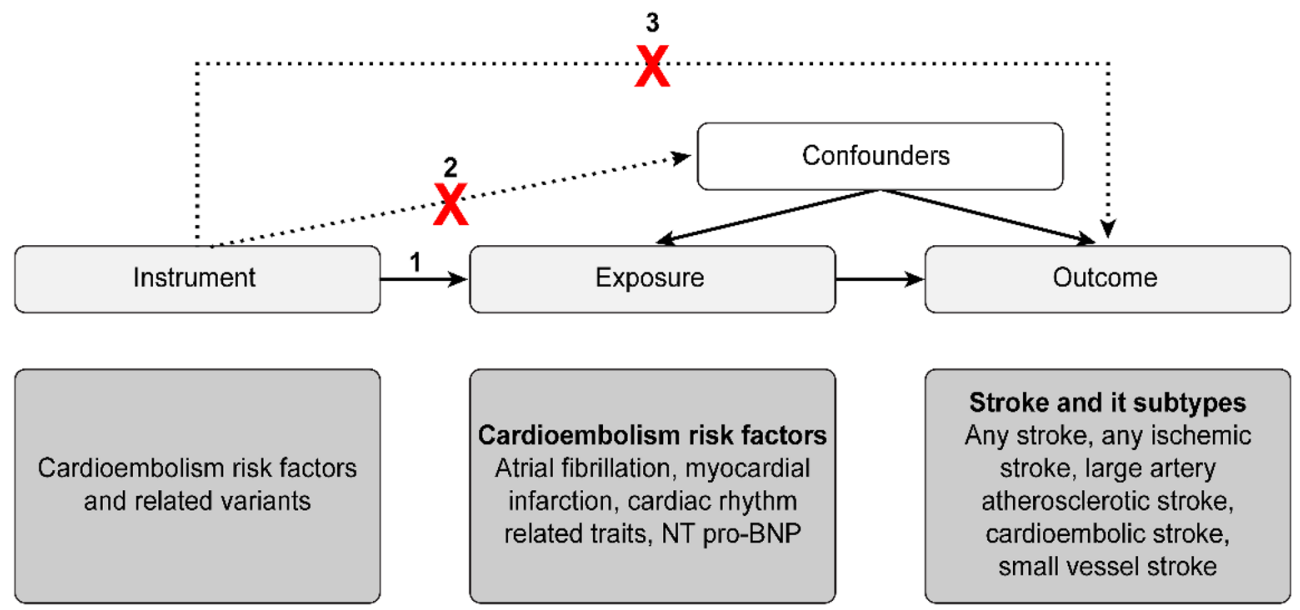

Figure 3. Schematic representation of Mendelian randomization analysis. Broken lines represent potential pleiotropic or direct causal effects between variables that would violate Mendelian randomization assumptions. SNP single-nucleotide polymorphism.

\begin{tabular}{|c|c|c|c|c|c|c|c|c|}
\hline \multirow[b]{2}{*}{ Traits } & \multicolumn{4}{|l|}{ Published GWAS } & \multicolumn{4}{|c|}{ Present MR study } \\
\hline & Reference & Sample size & $\begin{array}{l}\text { No. of significant } \\
\text { SNPs }\end{array}$ & r2 & $\begin{array}{l}\text { No. of SNPs } \\
\text { included }\end{array}$ & $\begin{array}{l}\text { Proxies used } \\
(\mathrm{r} 2>0.9)\end{array}$ & Power $^{\mathrm{a}}$ & $\mathbf{F}^{\mathrm{a}}$ \\
\hline $\mathrm{AF}$ & $\begin{array}{l}\text { Roselli et al., } \\
2018^{29}\end{array}$ & 65,556 & 104 & 0.15 & 104 & 0 & 1.00 & 4175 \\
\hline MI & $\begin{array}{l}\text { Nikpay et al., } \\
2015^{30}\end{array}$ & 43,676 & 26 & 0.04 & 25 & 0 & 0.65 & 177,222 \\
\hline RHR & $\begin{array}{l}\text { den Hoed et al., } \\
2013^{40}\end{array}$ & 134,251 & 68 & 0.02 & 62 & 0 & 0.06 & 8436 \\
\hline PTFV1 & $\begin{array}{l}\text { Christophersen } \\
\text { et al., } 2017^{31}\end{array}$ & 44,456 & 6 & 0.006 & 5 & 0 & 0.05 & 2496 \\
\hline PWD & $\begin{array}{l}\text { Christophersen } \\
\text { et al., } 2017^{31}\end{array}$ & 44,456 & 8 & 0.01 & 8 & 0 & 0.05 & 4176 \\
\hline NT-pro BNP & $\begin{array}{l}\text { Johansson et al., } \\
2016^{32}\end{array}$ & 18,624 & 3 & 0.003 & 3 & 0 & 0.05 & 1245 \\
\hline PR interval & $\begin{array}{l}\text { van Setten et al., } \\
2018^{34}\end{array}$ & 92,340 & 44 & 0.05 & 36 & 0 & 0.05 & 21,754 \\
\hline
\end{tabular}

Table 1. Genome-wide association studies and number of single-nucleotide polymorphisms used as instrumental variables in the Mendelian randomization analyses of cardioembolic risk factors in relationship to stroke and its subtypes. $A F$ atrial fibrillation, $M I$ myocardial infarction, $R H R$ resting heart rate, PTFV1 Pwave terminal force in lead V1, PWD P-wave duration. ${ }^{\mathrm{a}}$ Calculated for cardioembolic stroke.

\section{Methods}

Study design. The MR approach was based on the following three assumptions (Fig. 3$)^{28}$ : first, the genetic variant selected as instrumental variable is associated with cardioembolic risk factor; second, the genetic variant is not associated with any unmeasured confounders; and third, the genetic variant is associated with ischemic stroke only through cardioembolic risk factors, not through other pathways.

Cardioembolic risk factors. We considered cardioembolic risk factors that can be grouped under the following categories: cardiac disease related to CES, indices for left atrial abnormalities and other ECG indices that might be correlated with stroke. Within these categories, we focused on factors that were identified as having established or possible evidence for an association with ischemic stroke, especially CES.

Data sources. Summarized data for cardioembolic risk factors (effect size estimates and their standard errors) were available from published genome-wide association studies (GWAS). We identified genetic variants with genome-wide significance $\left(\mathrm{P}<5 \times 10^{-8}\right)$ associated with $\mathrm{AF}^{29}, \mathrm{MI}^{30}, \mathrm{PTFV} 1, \mathrm{PWD}^{31}$, NT-pro $\mathrm{BNP}^{32}$, resting heart rate (RHR) $)^{33}$ and PR interval ${ }^{34}$ (Table 1 and supplement Table 1 ).

For ischemic stroke and its subtypes, we collected summary statistics from the METASTROKE Collaboration $^{35}$. GWAS from the consortiums contributed a total of 67,162 stroke cases and 454,450 controls. A total of 6688 large-artery atherosclerotic stroke (LAS)s, 9006 CESs and 11,710 small vessel strokes (SVSs) were identified using the Trial of ORG 10,172 in Acute Stroke Treatment (TOAST) classification system. 
Because all analyses were based on publicly available summary statistics and not individual-level data, no patients were involved in the design of the study, and no ethical approval from an institutional review board was required.

Genetic variants. Single-nucleotide polymorphisms (SNPs) associated with cardioembolic risk factors were selected in the combined meta-analysis of the discovery and replication samples of 6 published GWASs (Table 1). Linkage disequilibrium (defined as $\mathrm{r}^{2}<0.01$ ) with other genetic variants was conducted to ensure independent genetic variants. The variant with the lowest $P$ value for association with the risk factor was selected when we encountered linkage disequilibrium. In instances where SNPs were not available in a data set because of poor imputation quality, we replaced them with proxy SNPs if available $\left(r^{2}>0.9\right)$.

Totally 104 SNPs were identified as associated with AF, 26 SNPs were identified as associated with MI, 68 SNPs were identified as associated with RHR, 6 SNPs were identified as associated with PTFV1, 8 SNPs were identified as associated with PWD, 3 SNPs were identified as associated with NT-pro BNP, and 44 SNPs were identified as associated with PR interval with genome wide significance $\left(\mathrm{p}<5 \times 10^{-8}\right)$, which explained $15 \%, 4 \%$, $2 \%, 0.6 \%, 1 \%, 0.3 \%, 5 \%$ variance of each exposure respectively. No linkage disequilibrium was found between SNPs. The other information of genetic variability was shown on Table 1.

Statistical analysis. We calculated the estimates of the cardioembolic risk factors on ischemic stroke and its subtypes using the inverse-variance weighted (IVW) method, which provides a combined estimate of the causal estimate from each SNP. IVW is considered conventional MR, since it is equivalent to a two-stage least squares or allele score analysis using individual-level data ${ }^{36}$. Furthermore, sensitivity analyses such as the weighted median, simple median, and MR-Egger regression were conducted ${ }^{37}$. The results are presented as odds ratios (ORs) and 95\% confidence intervals (CIs) per 10 units genetically predicted increase in each trait. The results for causal effects of diseases on cardioembolic risk factors are presented as beta \pm SE per unit higher in $\log$ odds of disease. MR-Egger analysis was used to evaluate the pleiotropy effects ${ }^{28}$. To investigate the influence of outlying and/or pleiotropic genetic variants, we performed a leave-one-out analysis in which we omitted one genetic variant in turn ${ }^{38}$. The strength of the genetic instruments was tested with the F-statistic methods ${ }^{39}$. All SNPs for the same trait showed a strong association (F-statistic $>10)$.

We conducted the statistical analysis using $\mathrm{R}$ version 3.3.3 ( $\mathrm{R}$ foundation). We prespecified a Bonferronicorrected significance threshold of $\mathrm{p}=0.00142$ (where $\mathrm{p}=0.05 / 35$ [seven exposures and five outcomes]) to adjust for multiple testing. Associations with $\mathrm{p}$ values between 0.05 and 0.00142 were considered suggestive evidence of a possible association.

Received: 3 March 2021; Accepted: 5 July 2021

Published online: 16 July 2021

\section{References}

1. Krishnamurthi, R. V. et al. Global and regional burden of first-ever ischaemic and haemorrhagic stroke during 1990-2010: Findings from the Global Burden of Disease Study 2010. Lancet Glob. Health 1(5), e259-e281 (2013).

2. Tian D., Yang Q., Dong Q., et al. Trends in stroke subtypes and vascular risk factors in a stroke center in China over 10 years. Sci. Rep. 8 (2018).

3. Yang Q., Churilov L., Fan D., et al. 1.4 times increase in atrial fibrillation-related ischemic stroke and TIA over 12 years in a stroke center. J. Neurol. Sci. 379, 1-6 (2017).

4. Kamel, H. et al. Tailoring the approach to embolic stroke of undetermined source: A review. JAMA Neurol. 76(7), 855-861 (2019).

5. Kamel, H. et al. Atrial fibrillation and mechanisms of stroke: Time for a new model. Stroke 47(3), 895-900 (2016).

6. He, J. et al. P-wave indices and risk of ischemic stroke: A systematic review and meta-analysis. Stroke 48(8), 2066-2072 (2017).

7. Longstreth W. T., Jr., Kronmal R. A., Thompson J. L., et al. Amino terminal pro-B-type natriuretic peptide, secondary stroke prevention, and choice of antithrombotic therapy. Stroke 44(3), 714-719 (2013).

8. Healey, J. S. et al. Recurrent stroke with rivaroxaban compared with aspirin according to predictors of atrial fibrillation: Secondary analysis of the NAVIGATE ESUS randomized clinical trial. JAMA Neurol. 76(7), 764-773 (2019).

9. Kamel, H. et al. P-wave Morphology and the risk of incident ischemic stroke in the multi-ethnic study of atherosclerosis. Stroke 45(9), 2786-2788 (2014).

10. Liu, X. et al. Resting heart rate and the risk of atrial fibrillation. Int. Heart J. 60(4), 805-811 (2019).

11. Lindgren, M. et al. Resting heart rate in late adolescence and long term risk of cardiovascular disease in Swedish men. Int. J. Cardiol. 259, 109-115 (2018)

12. Hu, L. et al. Associations between resting heart rate, hypertension, and stroke: A population-based cross-sectional study. J. Clin. Hypertens. (Greenwich) 21(5), 589-597 (2019).

13. Kwok, C. S. et al. Prolonged PR interval, first-degree heart block and adverse cardiovascular outcomes: A systematic review and meta-analysis. Heart (British Cardiac Society) 102(9), 672-680 (2016).

14. Smith, G. D. \& Ebrahim, S. "Mendelian randomization": Can genetic epidemiology contribute to understanding environmental determinants of disease?. Int. J. Epidemiol. 32(1), 1-22 (2003).

15. Sposato, L. A. et al. Diagnosis of atrial fibrillation after stroke and transient ischaemic attack: A systematic review and meta-analysis. Lancet Neurol. 14(4), 377-387 (2015).

16. Deitelzweig S. B., Ogbonnaya A., Berenson K., et al. Prevalence of stroke/transient ischemic attack among patients with acute coronary syndromes in a real-world setting. Hosp. Pract. (1995) 38(4), 7-17 (2010).

17. Merkler A. E., Diaz I., Wu X., et al. Duration of heightened ischemic stroke risk after acute myocardial infarction. J. Am. Heart Assoc. 7(22), e010782 (2018).

18. Merkler A. E., Sigurdsson S., Eiriksdottir G., et al. Association between unrecognized myocardial infarction and cerebral infarction on magnetic resonance imaging. JAMA Neurol. (2019).

19. Woodward, M. et al. The association between resting heart rate, cardiovascular disease and mortality: Evidence from 112,680 men and women in 12 cohorts. Eur. J. Prevent. Cardiol. 21(6), 719-726 (2014). 
20. Aune D., Sen A., o'Hartaigh B., et al. Resting heart rate and the risk of cardiovascular disease, total cancer, and all-cause mortalityA systematic review and dose-response meta-analysis of prospective studies. Nutr. Metab. Cardiovasc. Dis. (NMCD) 27(6), 504-517 (2017).

21. Grassi, G., Seravalle, G. \& Mancia, G. Sympathetic activation in cardiovascular disease: Evidence, clinical impact and therapeutic implications. Eur. J. Clin. Invest. 45(12), 1367-1375 (2015).

22. Martinez-Selles, M. et al. Should we anticoagulate patients at high risk of atrial fibrillation?. Rev. Esp. Cardiol. (English ed) 69(4), 374-376 (2016).

23. Kamel, H. et al. Association between left atrial abnormality on ECG and vascular brain injury on MRI in the Cardiovascular Health Study. Stroke 46(3), 711-716 (2015).

24. Okin, P. M. et al. Electrocardiographic left atrial abnormalities and risk of incident stroke in hypertensive patients with electrocardiographic left ventricular hypertrophy. J. Hypertens. 34(9), 1831-1837 (2016).

25. Llombart, V. et al. B-type natriuretic peptides help in cardioembolic stroke diagnosis: pooled data meta-analysis. Stroke 46(5), 1187-1195 (2015).

26. Kim, J. et al. The association between cerebral atherosclerosis and arterial stiffness in acute ischemic stroke. Atherosclerosis 219(2), 887-891 (2011).

27. Chan, Y. H. et al. PR interval prolongation in coronary patients or risk equivalent: Excess risk of ischemic stroke and vascular pathophysiological insights. BMC Cardiovasc. Disord. 17(1), 233 (2017).

28. Bowden, J., Davey, S. G. \& Burgess, S. Mendelian randomization with invalid instruments: Effect estimation and bias detection through Egger regression. Int. J. Epidemiol. 44(2), 512-525 (2015).

29. Roselli, C. et al. Multi-ethnic genome-wide association study for atrial fibrillation. Nat. Genet. 50(9), 1225-1233 (2018).

30. Nikpay, M. et al. A comprehensive 1,000 Genomes-based genome-wide association meta-analysis of coronary artery disease. Nat. Genet. 47(10), 1121-1130 (2015).

31. Christophersen I. E., Magnani J. W., Yin X., et al. Fifteen genetic loci associated with the electrocardiographic P wave. Circ. Cardiovasc. Genet. 10(4) (2017).

32. Johansson, A. et al. Genome-wide association and Mendelian randomization study of NT-proBNP in patients with acute coronary syndrome. Hum. Mol. Genet. 25(7), 1447-1456 (2016).

33. Eppinga, R. N. et al. Identification of genomic loci associated with resting heart rate and shared genetic predictors with all-cause mortality. Nat. Genet. 48(12), 1557-1563 (2016).

34. van Setten, J. et al. PR interval genome-wide association meta-analysis identifies 50 loci associated with atrial and atrioventricular electrical activity. Nat. Commun. 9(1), 2904 (2018).

35. Malik, R. et al. Multiancestry genome-wide association study of 520,000 subjects identifies 32 loci associated with stroke and stroke subtypes. Nat. Genet. 50(4), 524-537 (2018).

36. Burgess, S., Butterworth, A. \& Thompson, S. G. Mendelian randomization analysis with multiple genetic variants using summarized data. Genet. Epidemiol. 37(7), 658-665 (2013).

37. Yavorska, O. O. \& Burgess, S. MendelianRandomization: An R package for performing Mendelian randomization analyses using summarized data. Int. J. Epidemiol. 46(6), 1734-1739 (2017).

38. Burgess, S. \& Thompson, S. G. Interpreting findings from Mendelian randomization using the MR-Egger method. Eur. J. Epidemiol. 32(5), 377-389 (2017).

39. Burgess, S. \& Thompson, S. G. Avoiding bias from weak instruments in Mendelian randomization studies. Int. J. Epidemiol. 40(3), 755-764 (2011).

40. den Hoed, M. et al. Identification of heart rate-associated loci and their effects on cardiac conduction and rhythm disorders. Nat. Genet. 45(6), 621-631 (2013).

\section{Acknowledgements}

This study was supported by Beijing Municipal Science and Technology Commission (Grant numbers D141100000114005). We thank the MEGESTROKE Consortium for making data publicly available.

\section{Author contributions}

D.F. and T.H. conceived and designed the study. D.T. and Z.Z. contributed to the material preparation and data collection acquisition and analysis of data. D.T. wrote the manuscript. D.F. reviewed and edited the manuscript. All authors read and approved the manuscript.

\section{Competing interests}

The authors declare no competing interests.

\section{Additional information}

Supplementary Information The online version contains supplementary material available at https://doi.org/ 10.1038/s41598-021-93979-y.

Correspondence and requests for materials should be addressed to T.H. or D.F.

Reprints and permissions information is available at www.nature.com/reprints.

Publisher's note Springer Nature remains neutral with regard to jurisdictional claims in published maps and institutional affiliations.

(c) (i) Open Access This article is licensed under a Creative Commons Attribution 4.0 International License, which permits use, sharing, adaptation, distribution and reproduction in any medium or format, as long as you give appropriate credit to the original author(s) and the source, provide a link to the Creative Commons licence, and indicate if changes were made. The images or other third party material in this article are included in the article's Creative Commons licence, unless indicated otherwise in a credit line to the material. If material is not included in the article's Creative Commons licence and your intended use is not permitted by statutory regulation or exceeds the permitted use, you will need to obtain permission directly from the copyright holder. To view a copy of this licence, visit http://creativecommons.org/licenses/by/4.0/.

(C) The Author(s) 2021 